\title{
Vegetation Database Main-Kinzig + Bergland
}

\author{
Thomas Michl \& Stefan Huck
}

\begin{abstract}
This paper describes the structure and content of the Vegetation Database Main-Kinzig + Bergland (GIVD ID EU-DE-021). The database was initiated in 2004 to prepare a regional classification of the vegetation in the study region and to compile occurrence maps on the basis of individual relevés. It aims at collecting all available phytosociological relevés from the study region that is roughly defined by the mountain regions Spessart, Vogelsberg, Rhön and the adjacent lowlands (situated in southeastern Hesse and northwestern Bavaria). The main districts in the study region are Main-Kinzig, Wetterau, Vogelsberg, Fulda, Rhön-Grabfeld, Aschaffenburg and Main-Spessart. Presently, 1,562 of an estimated total of 20,000 relevés are available in the database, which is run offline with TURBOVEG software. Most of the relevés are from the Spessart and adjacent lowlands in the district Main-Kinzig. The most frequently recorded vegetation types are grassland and fringe communities. The data are used to prepare regional classifications by the authors. Proposals for joint analyses, e.g. supra-regional analyses and cooperation in large-scale classifications, are welcome.
\end{abstract}

Keywords: biodiversity informatics; Calluno-Ulicetea; Molinio-Arrhenatheretea; phytosociology; Spessart; Trifolio-Geranietea sanguinei; vegetation classification

\section{Vegetation Database Main-Kinzig + Bergland}

Scope: We aim at collecting all available phytosociological relevés of all vegetation types from the study region (Spessart, Vogelsberg, Rhön and adjacent lowlands; situated in southeastern Hesse and northwestern Bavaria).

Status: ongoing capture

Period: 1972-2010

Database manager(s): Thomas Michl (michl@buero-huck.de)

Owner: Dr. Huck Environmental Consultants Thomas Michl (michl@buero-huck.de) and Stefan Huck (private)

Web address: http://www.buero-huck.de/index.php?article_id=13

Availability: according to a specific agreement

Online upload: no

Online search: no

Database format(s): TURBOVEG Export format(s): TURBOVEG

Publication: [NA]

Plot type(s): normal plots

Non-overlapping plots: 1,562

Plot-size range: $0.12-450 \mathrm{~m}^{2}$

Total plot observations: 1,562

Estimate of existing plots: 20,000

Number of sources: 10

Completeness: $8 \%$

Valid taxa: [NA]

Countries: DE: $100.0 \%$

Forest: [NA] — Non-forest: [NA]

Guilds: all vascular plants: $100 \%$; bryophytes (terricolous or aquatic): $60 \%$; lichens (terricolous or aquatic): $60 \%$

Environmental data: altitude: $100 \%$; slope aspect: $36 \%$; slope inclination: $32 \%$; microrelief: $2 \%$; soil depth: $12 \%$; surface cover other than plants (open soil, litter, bare rock etc.): $12 \%$; other soil attributes: $3 \%$; land use categories: $12 \%$

Performance measure(s): cover: $100 \%$

Geographic localisation: GPS coordinates (precision $25 \mathrm{~m}$ or less): $35 \%$; point coordinates less precise than GPS, up to $1 \mathrm{~km}$ : $20 \%$; small grid (not coarser than $10 \mathrm{~km}): 30 \%$; political units or only on a coarser scale $(>10 \mathrm{~km}): 85 \%$

Sampling periods: 1970-1979: 0.2\%; 1980-1989: 18.6\%; 1990-1999: 28.8\%; 2000-2009: 47.4\%; 2010-2019: 5.0\% Information as of 2012-07-25; further details and future updates available from http://www.givd.info/ID/EU-DE-021

Thomas Michl* (michl@buero-huck.de), Stefan Huck (huck@buero-huck.de)

Dr. Huck Environmental Consultants, General-Colin-Powell-Str. 4, 63571 Gelnhausen, GERMANY

*Corresponding author 